\title{
The molecular mechanism of cytoadherence to placental or tumor cells through VAR2CSA from Plasmodium falciparum
}

\author{
Weiwei Wang ${ }^{1,2}$, Zhaoning Wang ${ }^{1,2}$, Xiuna Yang ${ }^{3}$, Yan Gao ${ }^{3}$, Xiang Zhang ${ }^{4}$, Long Cao ${ }^{1}$, Aguang Dai ${ }^{1,2}$, Jin Sun ${ }^{1,2}$, \\ Lei Sun ${ }^{4}$, Lubin Jiang ${ }^{1,2 \otimes}$, Zhenguo Chen ${ }^{4 凶}$ and Lanfeng Wang ${ }^{1,2 \bowtie}$
}

Dear Editor,

Pregnancy-associated malaria (PAM) threatened more than one million women and their infants in endemic regions in 2019. This resulted in maternal anemia, stillbirth, and infant death ${ }^{1,2}$. VAR2CSA encoded by a subfamily of var genes from Plasmodium falciparum ( $P$. falciparum) named as var2csa, plays a vital role in the cytoadherence of infected erythrocytes to the placenta ${ }^{3}$. Chondroitin sulfate A (CSA), which is displayed mostly on the surface of placental or tumor cells, has been recognized as a specific ligand for VAR2CSA ${ }^{4-7}$. However, the molecular mechanism of cytoadherence to placental or tumor cells through VAR2CSA remains elusive.

In this study, the VAR2CSA ectodomain from $P$. falciparum strain $3 \mathrm{D} 7 \quad(\sim 306 \mathrm{kDa}$, containing six Duffybinding-like (DBL) domains, N-terminal sequence (NTS), and multiple inter-domains (IDs) $)^{8}$ (Fig. 1a) was recombinantly expressed and purified using the Sf9 insect cell secretory system. The cryo-EM structures of VAR2CSA ectodomain and its complex with CSA were determined at a resolution of $3.6 \AA$ and $3.4 \AA$, respectively (Fig. 1b, c; Supplementary Figs. S1-S5 and Table S1). In line with the previously proposed $\operatorname{model}^{9}$, our structures showed that the core region is well-defined and covers

\footnotetext{
Correspondence: Lubin Jiang (Ibjiang@ips.ac.cn) or

Zhenguo Chen (ZhenguoChen@fudan.edu.cn) or

Lanfeng Wang (lanfwang@ips.ac.cn)

${ }^{1}$ The Center for Microbes, Development and Health, CAS Key Laboratory of Molecular Virology \& Immunology, Institut Pasteur of Shanghai, Chinese

Academy of Sciences, University of Chinese Academy of Sciences, Shanghai, China

${ }^{2}$ College of Life Sciences, University of Chinese Academy of Sciences, Beijing, China

Full list of author information is available at the end of the article

These authors contributed equally: Weiwei Wang, Zhaoning Wang, Xiuna

Yang, Yan Gao, Xiang Zhang.
}

NTS, DBL1X, DBL2X, ID2a, ID2b, DBL3X, ID3, and DBL4ع (Fig. 1b, c; Supplementary Fig. S6a). However, DBL5 $\varepsilon$ and DBL6e formed a flexible wing region. Compared with the apo structure, the densities of the wing region in the complex were significantly improved, making it feasible for the flexible fitting of both DBL5 $\varepsilon$ and DBL6e (Supplementary Fig. S4d). As for the core region, DBL2X and DBL4e stacked closely with ID2a, ID2b, and ID3, and formed the most stable core center, which served as a base for anchoring DBL3X and DBL1X at the top or bottom sites, respectively (Fig. 1b, c). Intriguingly, a highly basic pocket formed by DBL2X, DBL1X, NTS, and DBL4ع has been identified to accommodate a dodecasaccharide with six sulfated disaccharide repeats from CSA, which fitted well into the density map. Moreover, 16 residues were identified to be responsible for the direct interaction with 10/12 monosaccharides, except for the 6th and 11th units of CSA dodecasaccharide. Among them, there are nine, four, and three residues derived from DBL2X, DBL4 $\varepsilon$, and NTS, respectively. Furthermore, the nine residues of DBL2X were shown to interact directly with 7/ 10 monosaccharide units and thus may account for the major contribution to CSA binding (Fig. 1g; Supplementary Figs. S4e, f, S6b, c).

Owing to its high resolution and potential high stability in the core center, DBL4ع was used as an immobilized reference for the structural alignment between the VAR2CSA-CSA and VAR2CSA ectodomain (Fig. 1d-f). Interestingly, a significant conformational change in the core region was observed (Supplementary Movie S1). Firstly, the DBL1X moves closer to DBL4ع at more than $3.2 \AA$ (ranging from $3.2 \AA$ to $4.4 \AA$ ) for multi-helixes to facilitate NTS interaction with CSA and close the pocket (Fig. 1f). Secondly, there was a $1.8 \AA$ outward bend for the

\section{(c) The Author(s) 2021}

(c) (i) Open Access This article is licensed under a Creative Commons Attribution 4.0 International License, which permits use, sharing, adaptation, distribution and reproduction cc) in any medium or format, as long as you give appropriate credit to the original author(s) and the source, provide a link to the Creative Commons license, and indicate if changes were made. The images or other third party material in this article are included in the article's Creative Commons license, unless indicated otherwise in a credit line to the material. If material is not included in the article's Creative Commons license and your intended use is not permitted by statutory regulation or exceeds the permitted use, you will need to obtain permission directly from the copyright holder. To view a copy of this license, visit http://creativecommons.org/licenses/by/4.0/. 


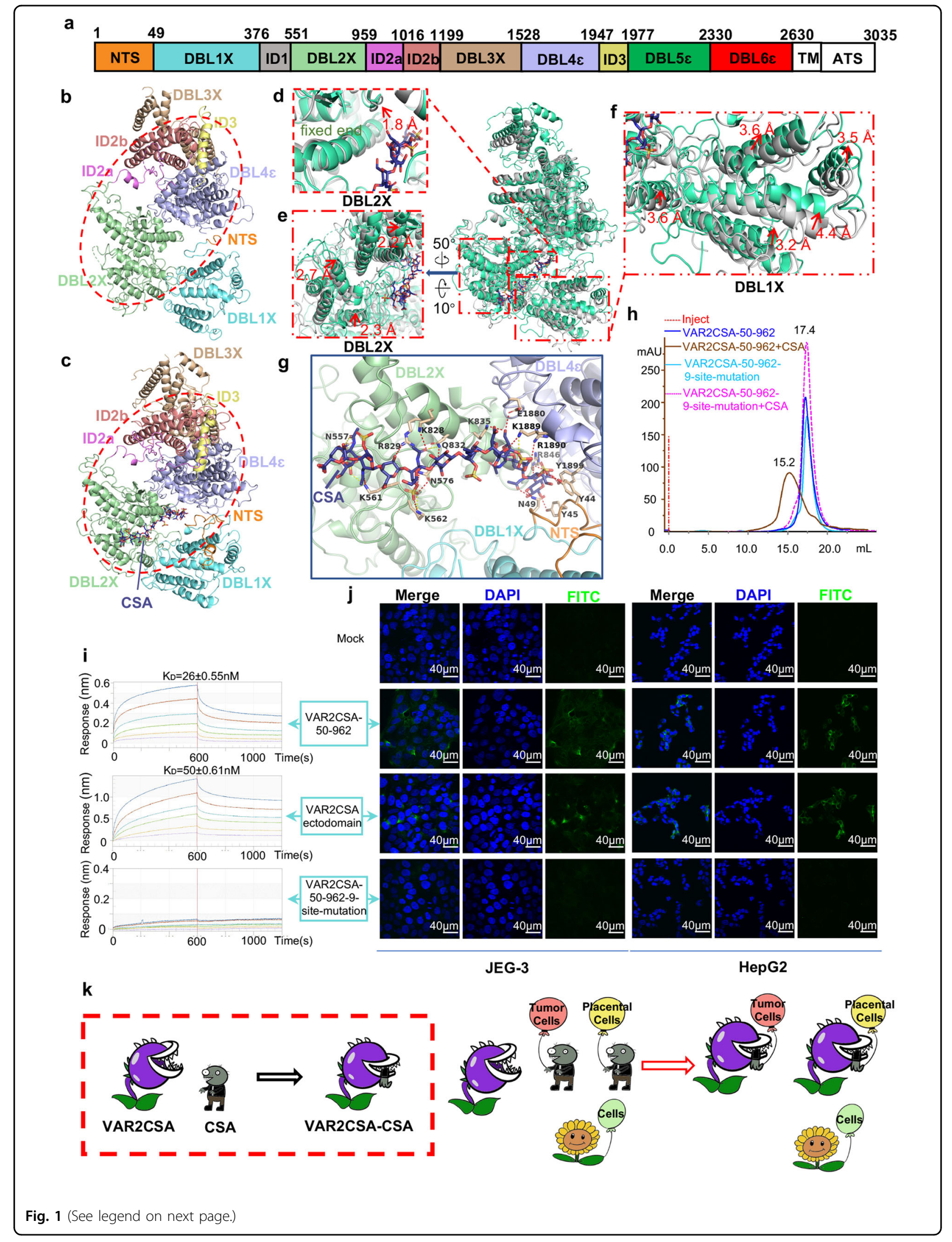


(see figure on previous page)

Fig. 1 Overall structures of VAR2CSA ectodomain. a Schematic sequence architecture of VAR2CSA of $P$. falciparum 3D7 strain. Domains reconstructed according to the cryo-EM density map were colored respectively. NTS, orange; DBL1X, aquamarine; DBL2X, pale green; ID2a, violet;

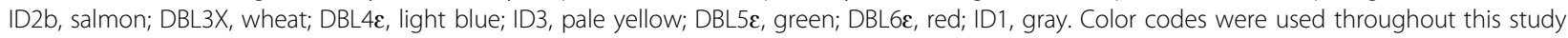
unless otherwise noted. $\mathbf{b}$ The ribbon diagram of apo VAR2CSA ectodomain core region. $\mathbf{c}$ The ribbon diagram of VAR2CSA-CSA core region. The core center was highlighted using red ovals in $\mathbf{b}$ and $\mathbf{c}$. $\mathbf{d}$-f Structural comparison between VAR2CSA-CSA with apo VAR2CSA ectodomain by

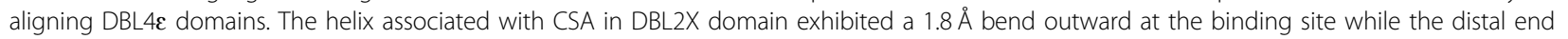
remained fixed (d). The surficial helixes in DBL2X moved about $2 \AA$ toward the CSA binding pocket (e). The DBL $1 X$ domain went through a significant conformational change at a distance of more than $3.2 \AA$ to close the CSA binding pocket $(\mathbf{f}) . \mathbf{g}$ Interactions between CSA and VAR2CSA were shown in the binding pocket. Interacting residues from NTS, DBL2X, and DBL4 $\varepsilon$ were highlighted individually. $\mathbf{h}$ Gel filtration chromatography of VAR2CSA50-962 and VAR2CSA-50-962-9-site-mutation (N557D, K561E, K562E, N576D, K828E, R829E, Q832E, K835E, R846E) in the presence or absence of CSA on a Superose 6 increase 10/300 GL column. i The binding kinetics of VAR2CSA ectodomain, VAR2CSA-50-962, and VAR2CSA-50-962-9-site-mutation were further assessed using Octet RED 96, and equilibrium constant $\left(K_{D}\right)$ values were calculated accordingly. $\mathbf{j}$ The binding activities of VAR2CSA-50962, VAR2CSA ectodomain, and VAR2CSA-50-962-9-site-mutation to JEG-3 cells or HepG2 cells were tested by immunofluorescence assay using confocal fluorescence microscopy, respectively. Green (stained with anti-His-FITC antibody) indicated VAR2CSA proteins; blue (DAPI) represented cell nuclei. $\mathbf{k}$ Model of VAR2CSA recognizing both placental cells and tumor cells. The cartoon exhibited the mechanism of VAR2CSA capturing the CSA ligand and showed the conformational change upon CSA binding in a red frame. The cartoon also illustrated the mechanism of VAR2CSA characteristically recognizing either placental cells or tumor cells by identifying the specific CSA displayed on the cell surface. VAR2CSA was drawn as cannibal plants in two states corresponding to CSA binding or not, respectively. CSA modifications were drawn as zombies and other glycolmodifications as sunflowers. Cells were depicted as balloons in different colors. Tumor cells, salmon; Placental cells, yellow; Normal cells, light green.

key CSA binding helix of DBL2X in the deep pocket (Fig. 1d). Lastly, the rest of DBL2X moved about $2 \AA$ inward to shrink the pocket (Fig. 1e).

Next, we aimed to identify the minimal structural elements required for CSA binding. First, the individual structures of four DBL domains (DBL1X, DBL2X, DBL3X, and $\mathrm{DBL} 4 \varepsilon$ ) in the core region are quite similar to each other, with the typical " $3+2$ " helix bundle shared (Supplementary Fig. S7). Sequence alignment showed that the nine key residues in DBL2X were rarely conserved within intramolecular DBL domains but highly conserved across various $P$. falciparum strains, suggesting a common CSA recognition mechanism dominated by DBL2X (Supplementary Figs. S8 and S9). Accordingly, two truncations, VAR2CSA-50-962 (DBL1X and DBL2X) and VAR2CSA-550-962 (DBL2X alone), were generated, and the CSA binding activity was evaluated using gel filtration chromatography. Similar to the VAR2CSA ectodomain, both VAR2CSA-50-962 and VAR2CSA-550-962 formed stable complexes with CSA (Supplementary Fig. S10). VAR2CSA-50-962 was selected as a representative for subsequent experiments due to its high yield. The nine key residues were mutated to $\mathrm{D}$ or $\mathrm{E}$ to obtain VAR2CSA-50-962 N557D, K561E, K562E, N576D, K828E, R829E, Q832E, K835E, and R846E (VAR2CSA-50962-9-site-mutation).

Both VAR2CSA-50-962 and VAR2CSA-50-962-9-sitemutation in the presence or absence of CSA were subjected to analysis using gel filtration chromatography. Interestingly, the CSA binding activity of the VAR2CSA50-962-9-site-mutation was totally disrupted in comparison with VAR2CSA-50-962 (Fig. 1h). Additionally, Octet RED 96 was used to test the CSA binding affinities of the VAR2CSA ectodomain, VAR2CSA-50-962, and VAR2CSA-50-962-9-site-mutation. In accordance with the results of chromatography above, the VAR2CSA ectodomain and VAR2CSA-50-962 exhibited a strong affinity for CSA, while the VAR2CSA-50-962-9-sitemutation showed no binding activity (Fig. 1i).

Significantly, it was further verified that the 9-sitemutation could eliminate the binding activity of VAR2CSA to placental cells or tumor cells using confocal fluorescence microscopy. Briefly, $500 \mathrm{nM}$ individual protein (VAR2CSA ectodomain, VAR2CSA-50-962, or VAR2CSA50-962-9-site-mutation) was incubated with cells (JEG-3 and HepG2 representing placental cells or tumor cells, respectively) pre-seeded on a slide. After thorough washing, the fluorescent-labeled anti-His monoclonal antibody was added to stain the retarded proteins, while DAPI was applied to identify cell nuclei. The slides were analyzed using an Olympus FV1200 laser scanning confocal microscope. Compared to the controls (no proteins added), VAR2CSA50-962 and VAR2CSA ectodomain could specifically bind to JEG-3 cells and HepG2 cells, and no obvious difference in the binding affinity was observed between the two proteins, while the cell-binding activity of the VAR2CSA-50-962-9site-mutation was eliminated (Fig. 1j). This is in line with the biochemistry results described above. Taken together, the 9site-mutation in DBL2X can eliminate the binding activity of VAR2CSA to placental cells or tumor cells under the conditions tested.

Recently, the cryo-EM structures of the VAR2CSA ectodomain from $P$. falciparum strain FCR3 and VAR2CSA ectodomain from $P$. falciparum strain NF54 complexed with CSA were reported ${ }^{10}$. Meanwhile, another study also revealed the cryo-EM structures of the VAR2CSA ectodomain from $P$. falciparum strain FCR3 and its complex with placental chondroitin sulfate ${ }^{11}$. Uniquely, our structural study showed that CSA binding induced significant conformational changes to secure ligand binding, which was not reported by these two works ${ }^{10,11}$. The conformation in the 
former work ${ }^{10}$ is different from ours, which might be due to the fact that VAR2CSA proteins in apo structure and complex structure are from two different $P$. falciparum strains. In the other study ${ }^{11}$, FCR3 VAR2CSA shared a sequence identity of $79 \%$ with 3D7 VAR2CSA used in our study, but no ligand density was reported in their complex structure. Most importantly, beyond the structural study, we also generated VAR2CSA fragments and 9-site mutations, and evaluated their binding activity to either isolated CSA or placental and tumor cell lines. These results clearly showed that the 9-site-mutation in DBL2X eliminated the CSA binding activity in vitro and disrupted its binding to both placental and tumor cells.

In summary, we have elucidated the detailed molecular mechanism of cytoadherence to placental or tumor cells through VAR2CSA, presented in the working model (Fig. $1 \mathrm{k})$. Our results may facilitate antigen design for the PAM vaccine development and improve the drug delivery systems targeting both placenta and tumor cells.

\section{Acknowledgements}

We would like to pay an exceptional tribute to the staff members of the CryoEM facility of Fudan University (Fenglin Campus) and the Bio-Electron Microscopy Facility of ShanghaiTech University for their generous support in cryo-sample preparation and data collection. We thank the staffs, particularly Jie Li and Lei Zhang, of the Large-scale Protein Preparation System at the National Facility for Protein Science in Shanghai (NFPS), Zhangjiang Lab for providing technical support and assistance in data collection and analysis using Octet RED 96. We also thank Dr. Yuan Tian and the other members of the scientific core facility in Institut Pasteur of Shanghai, Chinese Academy of Science, for their help in negative staining TEM and confocal fluorescence microscopy. This work was funded by the National Key R\&D Program of China (2018YFA0507303, 2017YFC0840300), the Strategic Priority Research Program of CAS (XDB29010205), Shanghai Municipal Science and Technology Major Project (2019SHZDZX02), and the National Natural Science Foundation of China (NSFC) $(31770816,82025023,31771455,31970146)$.

\footnotetext{
Author details

'The Center for Microbes, Development and Health, CAS Key Laboratory of Molecular Virology \& Immunology, Institut Pasteur of Shanghai, Chinese Academy of Sciences, University of Chinese Academy of Sciences, Shanghai, China. ${ }^{2}$ College of Life Sciences, University of Chinese Academy of Sciences, Beijing, China. Institute for Advanced Immunochemical Studies and School of Life Science and Technology, ShanghaiTech University, Shanghai, China. ${ }^{4}$ Shanghai Fifth People's Hospital, Fudan University, and Shanghai Key Laboratory of Medical Epigenetics, International Co-laboratory of Medical Epigenetics and Metabolism (Ministry of Science and Technology), Institutes of Biomedical Sciences, Fudan University, Shanghai, China
}

\section{Author contributions}

L.W., Z.C., and L.J. conceived, designed, and coordinated this project; W.W. and L.C. expressed and purified the N-terminal domain; W.W. prepared the rest samples containing ectodomain, complex, and mutations; W.W., J.S., and Z.W. carried out the Octet RED 96 and confocal fluorescence microscopy experiments; Z.C., L.S., X.Y., Z.W., Y.G., and X.Z. prepared the cryo-samples and collected cryo-EM data; Z.C., Z.W., Y.G., and A.D. processed cryo-EM data; L.W. and Z.W. built and refined the structure models; the manuscript was written by L.W., W.W., Z.W., L.S., Z.C., X.Y., and Y.G., all authors discussed the experiments and results, read, and approved the manuscript.

\section{Competing interests}

The authors declare no competing interests.

\section{Publisher's note}

Springer Nature remains neutral with regard to jurisdictional claims in published maps and institutional affiliations.

Supplementary information The online version contains supplementary material available at https://doi.org/10.1038/s41421-021-00324-8.

Received: 9 July 2021 Accepted: 15 August 2021

Published online: 19 October 2021

\section{References}

1. Desai, $\mathrm{M}$. et al. Epidemiology and burden of malaria in pregnancy. Lancet Infect. Dis. 7, 93-104 (2007).

2. World Malaria Report 2019. xxxix, 185 p. World Health Organization (2019).

3. Salanti, A. et al. Selective upregulation of a single distinctly structured var gene in chondroitin sulphate A-adhering Plasmodium falciparum involved in pregnancy-associated malaria. Mol. Microbiol. 49, 179-191 (2003).

4. Gamain, B. et al. Identification of multiple chondroitin sulfate A (CSA)-binding domains in the var2CSA gene transcribed in CSA-binding parasites. J. Infect. Dis. 191, 1010-1013 (2005).

5. Singh, K. et al. Structure of the DBL3x domain of pregnancy-associated malaria protein VAR2CSA complexed with chondroitin sulfate A. Nat. Struct. Mol. Biol. 15, 932-938 (2008).

6. Bir, N. et al. Immunogenicity of duffy binding-like domains that bind chondroitin sulfate $\mathrm{A}$ and protection against pregnancy-associated malaria. Infect. Immun. 74, 5955-5963 (2006).

7. Salanti, A. et al. Targeting human cancer by a glycosaminoglycan binding malaria protein. Cancer Cell 28, 500-514 (2015).

8. Srivastava, A. et al. VAR2CSA minimal CSA binding region is located within the N-terminal region. PLoS One 6, e20270 (2011).

9. Bewley, M. C., Gautam, L., Jagadeeshaprasad, M. G., Gowda, D. C. \& Flanagan, J. M. Molecular architecture and domain arrangement of the placental malaria protein VAR2CSA suggests a model for carbohydrate binding. J. Biol. Chem. 295, 18589-18603 (2020).

10. $\mathrm{Ma}, \mathrm{R}$. et al. Structural basis for placental malaria mediated by Plasmodium falciparum VAR2CSA. Nat. Microbiol. 6, 380-391 (2021).

11. Wang, $K$. et al. Cryo-EM reveals the architecture of placental malaria VAR2CSA and provides molecular insight into chondroitin sulfate binding. Nat. Commun. 12, 2956-2956 (2021). 